\title{
Morphologic Features of Melanocytes, Pigmented Keratinocytes, and Melanophages by In Vivo Confocal Scanning Laser Microscopy
}

\author{
Klaus J. Busam, M.D., Carlos Charles, M.D., Grace Lee, M.D., Allan C Halpern, M.D. \\ Department of Pathology (KJB, GL) and Dermatology Service (CC, ACH), Department of Medicine, \\ Memorial Sloan-Kettering Cancer Center, New York, New York
}

Confocal scanning laser microscopy (CSLM) represents a novel imaging technique for in vivo microscopic analysis of skin lesions at a level of resolution that allows morphologic analysis of microanatomic structures. We investigated the feasibility of recognizing the cellular constituents of pigmented skin lesions, such as pigmented keratinocytes, melanocytes, and melanophages, by CSLM. Fifteen pigmented lesions (five pigmented seborrheic keratoses, and 10 compound melanocytic nevi) from 15 patients were studied, as well as normal skin. After the clinical lesions were imaged by CSLM, they were biopsied or excised for examination by conventional histology for comparison of the morphologic features. In images obtained by CSLM, pigmented keratinocytes were seen as polygonal cohesive cells with variably bright granular cytoplasm. Melanocytes appeared as bright round, oval, fusiform, or dendritic cells. The architectural growth pattern of melanocytes could be analyzed. Melanocytes were identified by their nested growth pattern as aggregates of bright round to oval structures at the dermoepidermal junction or in the superficial dermis. Melanocytes were also recognizable as single cells along the dermoepidermal junction, usually separated from each other by a variable number of keratinocytes. Melanophages appeared as large bright plump cells with ill-defined cytoplasmic borders, usually located around or near vessels of the superficial dermis. Our results demonstrate that the cellular constituents of pigmented lesions can be recognized by CSLM. This technique sets a new paradigm for noninvasive quasihistologic examina-

Copyright $(2001$ by The United States and Canadian Academy of Pathology, Inc.

VOL. 14, NO. 9, P. 862, 2001 Printed in the U.S.A.

Date of acceptance: April 18, 2001

This work was supported by Lucid, Inc., Henrietta, New York.

Address reprint requests to: Klaus J. Busam, M.D., Department of Pathol-

ogy, Memorial Sloan-Kettering Cancer Center, 1275 York Avenue, New

York, NY 10021; e-mail: busamk@mskcc.org; fax: 212-717-3203. tion of pigmented lesions in vivo and merits further evaluation for diagnostic use.

KEY WORDS: Confocal microscopy, Keratinocytes, Melanocytes, Melanophages.

Mod Pathol 2001;14(9):862-868

One of the most challenging problems in clinical dermatology is the early detection of malignant melanoma. All noninvasive melanomas and the majority of thin invasive melanomas are cured by complete excision. Delayed recognition of melanoma needs to be avoided because it puts the patient at risk for death from disease once the tumor has progressed to competence for metastasis. Early detection of melanoma currently relies on the critical macroscopic visual analysis of a new or changing pigmented lesion and subsequent biopsy or excision for diagnosis and treatment.

The clinical diagnostic accuracy of physicians, however, is less than perfect (1). The search for new methods that may aid in the early detection of melanomas has led to the development of novel imaging modalities, such as computer-assisted image analysis (2), dermoscopy (3), and confocal scanning laser microscopy (CSLM; 4, 5). Of these techniques, CSLM offers the highest level of resolution approaching histologic detail. It is currently being explored for the diagnosis of a variety of skin lesions (6-13), including melanoma (14).

Because assessment of a lesion by CSLM relies on the visualization of microanatomic structures, interpretation of images obtained by CSLM represents in principle a histopathologic evaluation. However, little is known about this novel technique, according to the pathology literature. Although CSLM has been pioneered by dermatologists, it is important for pathologists to get involved in the evaluation of this technique and to share their experience in its use. The more lesions are examined in parallel by CSLM and conventional 
histology, the better we can judge the potential and limitations of this promising new technique.

As a first step to exploring the potential use of CSLM in the analysis of pigmented lesions, we sought to determine whether the cellular constituents of pigmented lesions, in other words, melanocytes, pigmented keratinocytes, and melanophages, can be recognized by CSLM. In this study, we describe the morphologic features of pigmented cells by CSLM in comparison with conventional histology.

\section{MATERIALS AND METHODS}

\section{Subjects}

Fifteen patients were recruited from the pigmented lesion clinic at Memorial Sloan-Kettering Cancer Center. They gave informed consent for examination of their lesions by CSLM.

\section{Confocal Scanning Laser Microscopy}

Confocal imaging was performed with a commercially available, near-infrared, reflectance confocal laser scanning microscope (Vivascope 1000, Lucid, Inc., Henrietta, NY). The instrument uses a diode laser at $830 \mathrm{~nm}$ with a power of $<35 \mathrm{~mW}$ at tissue level. A $30 \times$ water-immersion objective lens of numerical aperture 0.9 was used with either water (refractive index, 1.33) or gel (refractive index, 1.3335) as an immersion medium. It images with a spatial resolution of 0.5 to $1.0 \mu \mathrm{m}$ in the lateral dimension and 4 to $5 \mu \mathrm{m}$ in the axial dimension. An automated stepper was used to obtain a grid of 16 contiguous horizontal images in $<20$ seconds to construct a montage image with an effective in vivo field of view of $1.6 \times 2.0 \mathrm{~mm}$. All images obtained by CSLM in this study correspond to sections in the horizontal plane.

\section{Histopathology}

Punch biopsies or excisions were fixed in formalin and embedded in paraffin. After routine processing, slides were stained with hematoxylin and eosin. In selected cases of clinically homogeneously appearing large lesions, a portion of the lesion was punched out and sectioned en face, in other words, in the horizontal plane, whereas the remainder of the excision was conventionally sectioned in serial vertical planes.

After histologic examination, the lesions of the 15 patients were classified as follows: five pigmented seborrheic keratoses and 10 compound melanocytic nevi. Eight nevi were atypical nevi (nevi with architectural disorder and atypia, "dysplastic" nevi). Two nevi were congenital compound nevi.

\section{RESULTS}

Keratinocytes and Melanophages in Normal Skin and Pigmented Seborrheic Keratoses

In normal skin, keratinocytes of the basilar and spinous cell layer appeared as polygonal cells (Fig. 1). Their size ranged from 10 to $20 \mu \mathrm{m}$ in greatest dimension. They formed a cohesive honeycomb pattern on cross-sections. The intercellular junctions appeared as bright lines. The nuclei corresponded to dark oval or round structures. The cytoplasm showed variable amounts of bright granular material. The brightest signals within the entire epidermis were usually obtained at the level of the granular cell layer.

Hyperpigmented basal layer keratinocytes were also brighter than the cells of the spinous cell layer. Pigmented keratinocytes at the dermoepidermal junction of a seborrheic keratosis are illustrated in an optical section by CSLM (horizontal plane) in Figure 2A. Cohesive polygonal cells with variably bright cytoplasm formed rings around papillae. The bright granular material in the cytoplasm of basilar keratinocytes was often eccentrically located at the site, oriented away from the papillae and toward the spinous cell layer. Some dermal papillae contained dark round or canalicular structures corresponding to capillaries. The corresponding conventional histology of a section in the vertical plane is illustrated in Figure 2B. Focally, clusters of melanophages were present in the papillary dermis, as illustrated in Figure 2C (confocal image; horizontal plane) and Figure 2D (conventional image; horizontal plane). In the confocal image, melanophages appeared as plump, bright cells with ill-defined cytoplasmic borders.

Five of the 15 examined pigmented lesions of this series were histologically diagnosed as pigmented

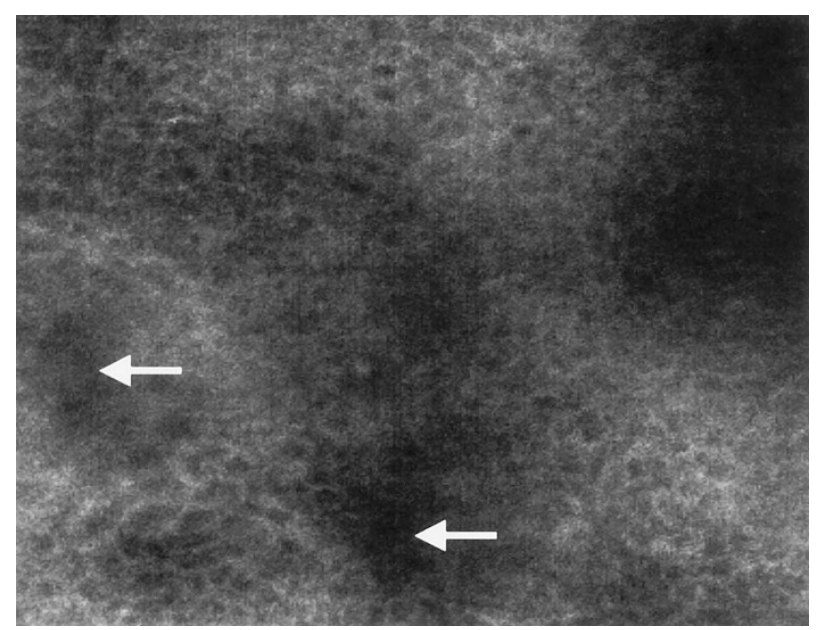

FIGURE 1. Normal skin. Keratinocytes form a honeycomb pattern of cohesive polygonal cells. Intercellular junctions appear bright. Dark spaces (arrows) indicate peaks of dermal papillae. 

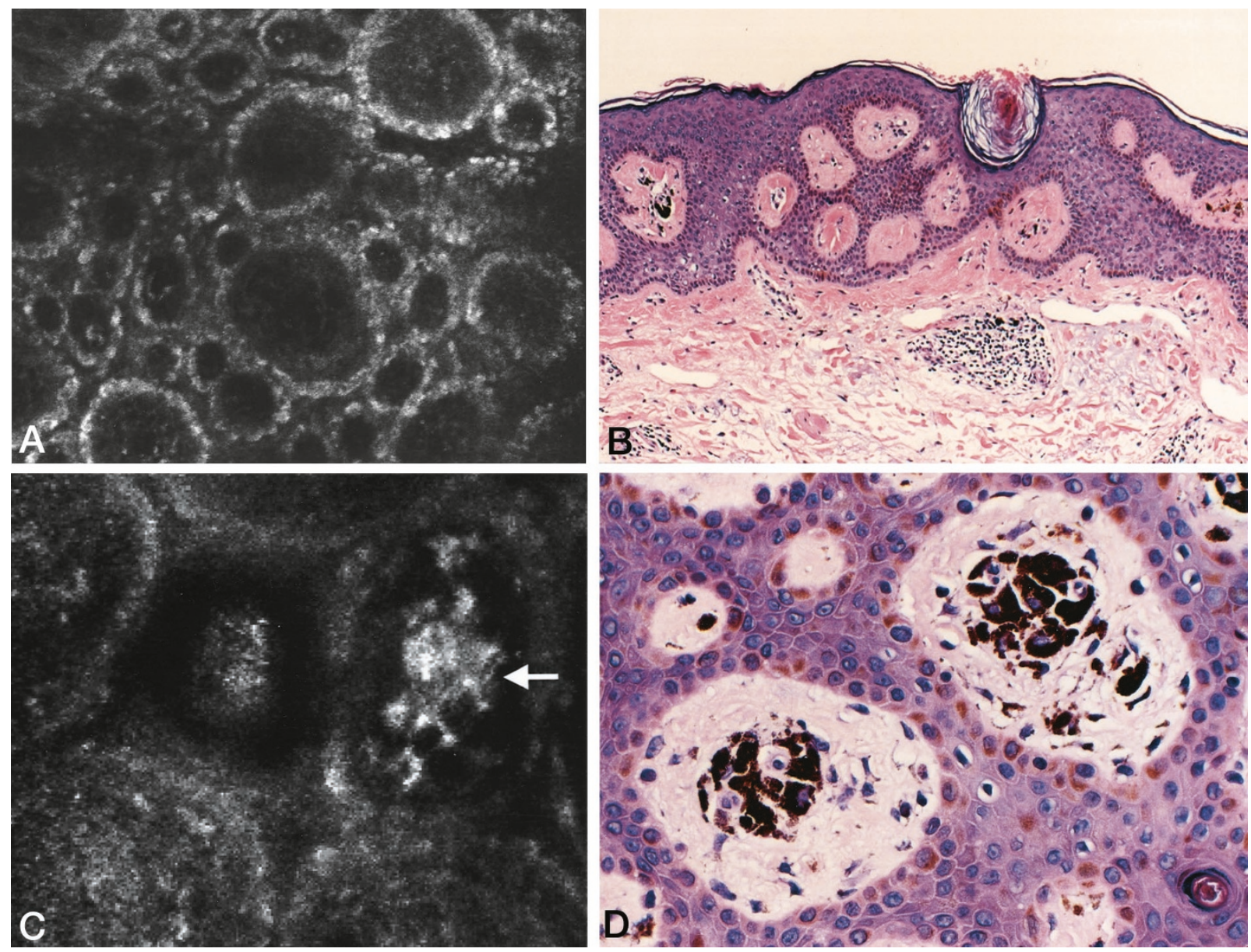

FIGURE 2. Keratinocytes and melanophages in a pigmented seborrheic keratosis. A, optical section obtained by confocal scanning laser microscopy (CSLM; horizontal plane); bright polygonal cells form rings around dermal papillae. Dark canalicular structures are seen in papillae, corresponding to blood vessels. B, hematoxylin and eosin (H\&E)-stained section (shown in conventional vertical plane) of the lesion. C, dermal papillae with melanophages (arrow) as seen in optical section by CSLM (in horizontal plane). D, focally, clusters of melanophages are present in dermal papillae (H\&E-stained section in horizontal plane).

seborrheic keratosis. In these three cases, confocal microscopy suggested the diagnosis of a probable pigmented keratosis by revealing a hyperplastic epidermis with many bright keratinocytes and no associated melanocytic proliferation.

\section{Melanocytes and Melanocytic Nevi}

Melanocytes were usually round or oval, but fusiform and dendritic shapes were also recognized (Fig. 3). Although the size and shape of their cytoplasm could be assessed, nuclear detail could not be evaluated at the level of magnification used in this study. As illustrated for a nevus in Figure 4, a nested growth pattern of melanocytes could be recognized by CSLM in the superficial dermis. The more melanin pigment the cells contained, the brighter they appeared under CSLM. Distinct cellular outlines were usually not seen within nests. Typically, the bright signals of individual cells fused and formed a spherical structure of variable size and shape with no further cellular detail. However, by reducing laser power and by using zooming op-

\section{Morphology of Melanocytes by CSLM}
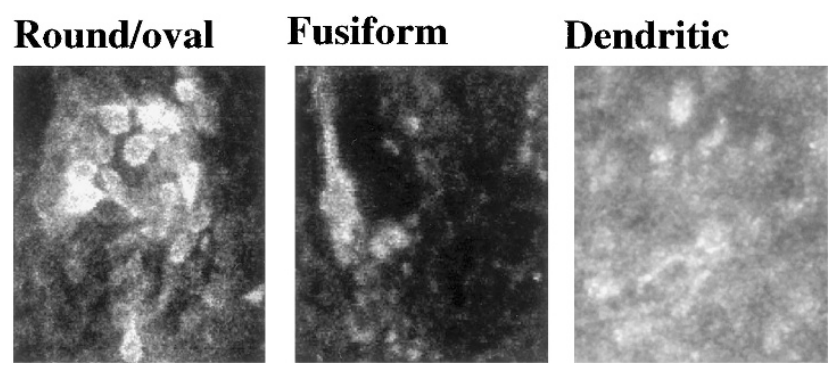

FIGURE 3. Morphology of individual melanocytes in optical sections obtained by confocal scanning laser microscopy.

tions, vague cellular outlines could occasionally be detected by CSLM even within nests.

For the distinction of junctional from dermal nests, examination of multiple horizontal sections in various vertical planes was necessary. This is best done in video mode or in serial horizontal optical sections at different depths from the skin surface. 


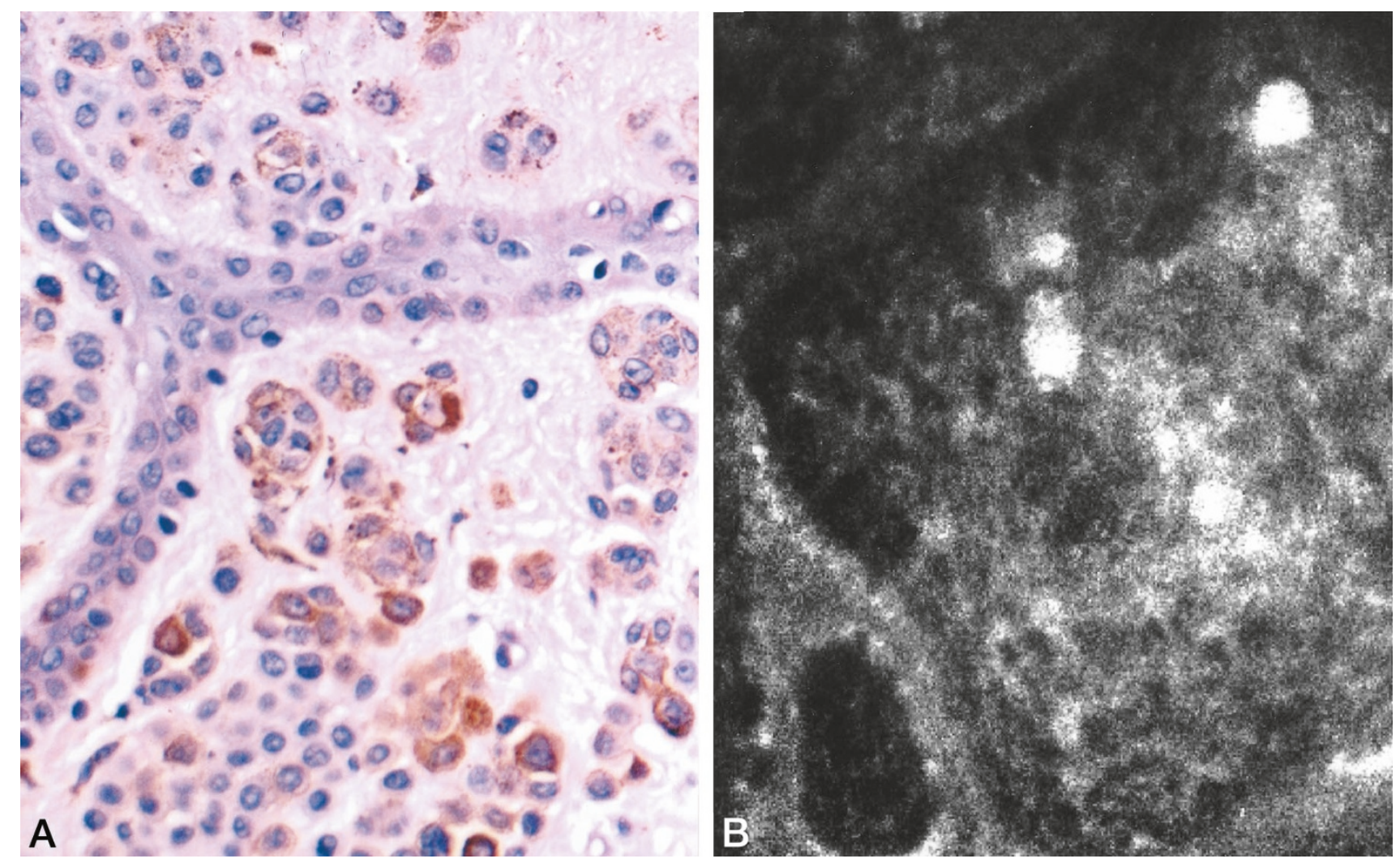

FIGURE 4. Compound melanocytic nevus. A, hematoxylin and eosin-stained section shown in horizontal plane at the level of the dermoepidermal junction and superficial dermis. B, optical section by confocal scanning laser microscopy (horizontal plane) corresponding to the conventional histology section shown in A.

An example of three sections at different depths is illustrated in Figure 5. At the level of the granular cells layer (Fig. 5A), keratinocytes appeared very bright. The background became increasingly darker with depth towards the dermoepidermal junction (Fig. 5B) and superficial dermis (Fig. 5C). Bright clusters of round or oval structures represented nests of melanocytes.

Of the 10 examined nevi, 8 were dysplastic nevi and 2 were congenital nevi. The eight dysplastic nevi differed from the congenital compound nevi by a more complex epidermal network, a more prominent single-cell growth of melanocytes at the dermoepidermal junction along elongated rete ridges, and a greater variation in the size and shape of nests with fusion of nests.

The morphologic features of pigmented keratinocytes, melanocytes, and melanophages under CSLM are summarized in Table 1.

\section{DISCUSSION}

CSLM is a novel imaging technique that allows in vivo examination of the epidermis and superficial dermis at a resolution approaching histologic detail $(4,5)$. It sets a new paradigm of instant noninvasive quasihistologic examination of skin lesions in vivo (15). Reflectance CSLM operates by detecting single back-scattered photons from the illuminated infocus plane of interest (16-18). High-resolution optical sections are achieved by means of a pinhole placed in an optical conjugated plane in front of the detector that rejects photons coming from out-offocus planes. Contrast in confocal images is provided by refraction index differences of organelles and other microstructures from the background. This means that highly refractile structures appear white on screen, whereas low-refractile structures appear dark gray to black $(4,5,18)$. This technique has been used to image keratinocytic and inflammatory skin lesions (6-13) and is currently also being investigated for the diagnosis of pigmented skin lesions (14).

Melanin pigment has previously been found to provide a natural contrast for confocal scanning (4). Its presence results in a bright white image signal, which illuminates the cytoplasm of melanincontaining cells. However, little is known about the cellular characteristics of individual pigmented cells by CSLM.

As our results illustrate, melanocytes can be recognized under CSLM as bright round to oval or elongated and fusiform structures with or without processes of various lengths. Melanocytes can be seen as solitary units or nests positioned at the dermoepidermal junction. Within nests, distinct 
TABLE 1. Morphologic Features of Pigmented Cells by Confocal Scanning Laser Microscopy

\begin{tabular}{|c|c|c|c|}
\hline Cell Type & Cell Shapes & Cell Arrangement & Signal Intensity \\
\hline Keratinocytes & Polygonal & $\begin{array}{l}\text { Cohesive cells; } \\
\text { honeycomb pattern }\end{array}$ & $\begin{array}{l}\text { Variably bright granular cytoplasm; } \\
\text { often eccentric signal in basal cells }\end{array}$ \\
\hline Melanocytes & $\begin{array}{l}\text { Round or oval } \\
\text { Fusiform } \\
\text { Dendritic }\end{array}$ & $\begin{array}{l}\text { Solitary units or nests in } \\
\text { epidermis or dermis }\end{array}$ & $\begin{array}{l}\text { Variably bright cytoplasm; usually } \\
\text { diffuse signal }\end{array}$ \\
\hline Melanophages & Oval or stellate & $\begin{array}{l}\text { Solitary cells or loose } \\
\text { aggregates of cells in } \\
\text { dermal papillae }\end{array}$ & $\begin{array}{l}\text { Variably bright cells with fuzzy cell } \\
\text { borders }\end{array}$ \\
\hline
\end{tabular}

cellular outlines are usually not detectable. Pigmented keratinocytes, on the other hand, appear as polygonal bright structures in cohesive aggregates with distinct cellular outlines. Above the basal cell layer, the network of visible cellular outlines of keratinocytes forms a honeycomb pattern. In basilar keratinocytes, the bright signal corresponding to pigmentation is often eccentrically positioned away from the dermal papillae (Fig. 2B). In melanocytes, the signal intensity is usually present throughout the entire cytoplasm in a uniform pattern (Figs. 3-5).

Although individual bright structures of the epidermis seen on confocal images can usually be identified as pigmented keratinocytes or melanocytes, it is often necessary to use architectural context in addition to the cellular morphologic features to identify the correct cell type. Without knowledge of architectural context, such as the location of a bright structure at the dermoepidermal junction and its separation from other such structures by darker polygonal cells, it would many times not be possible to correctly distinguish melanocytes lacking detectable dendritic processes from keratinocytes.

Melanophages are best recognized by their solitary distribution around papillary dermal capillaries. Their cell size is usually larger than that of individual melanocytes, and the bright granular cytoplasmic signal, often coarser.

Our results on normal skin are in agreement with those in previous reports $(4,5)$. When examining the epidermis from its top towards the superficial dermis, the stratum corneum produces the first bright image because of specular reflection caused by refractive index mismatch between the microscope immersion medium (water) and the skin (4, $5)$. Keratinocytes of the granular cell layer containing keratohyalin granules give a very bright signal in confocal images. Keratinocytes of the spinous and basal cell layer usually provide less contrast: although intercellular connections are well recognizable, the cytoplasm and especially nuclei appear dark. However, as illustrated in our study, pigmented keratinocytes are bright in confocal images depending on the amount of melanin pigment (Fig. 2).

Near-infrared CSLM is currently an investigational tool approved by the Food and Drug Admin- istration for human use. Although we have demonstrated herein that pigmented keratinocytes, melanocytes, and melanophages can be recognized by CSLM, it remains to be seen how sensitive the detection is for various pigmented lesions and to what extent specific diagnoses can be rendered or at least suspected by CSLM. At its current state of technology, CSLM has two major limitations compared with conventional histology. First, its level of resolution is inferior to that of conventional histology. Although cell size and shape can be determined by CSLM, nuclear features, such as chromatin patterns, nuclear contours, and nucleoli, cannot be evaluated. Second, CSLM can assess microanatomic structures only to a depth of approximately $300 \mu \mathrm{m}^{4}$. Thus, processes in the reticular dermis cannot be examined.

Nonetheless, CSLM merits evaluation for use as a screening tool for the examination of skin lesions, including pigmented lesions. As we have demonstrated, CSLM allows the recognition and position of melanocytes within the epidermis. Therefore, it may allow immediate recognition of a pigmented lesion as melanocytic in origin. This is of particular interest for the distinction of pigmented keratoses or solar lentigines from melanoma. In the five patients in this study with pigmented seborrheic keratoses, there was clinical concern about the possibility of malignant melanoma. Confocal microscopy failed to detect a melanocytic proliferation and suggested a pigmented keratosis. Because CSLM is currently an investigational tool, a biopsy was needed to confirm the diagnosis by conventional light microscopy. If future studies on a large number of lesions establish good correlation between CSLM and conventional histology in the distinction of a pigmented keratosis from a melanocytic neoplasm, the use of CSLM may eventually be of great benefit. It may spare some patients a biopsy procedure and save time and costs.

The potential diagnostic use of CSLM may not be limited to the basic distinction between a melanocytic and nonmelanocytic lesion. Once melanocytes are recognized, the ability to analyze their growth pattern within the epidermis should in principle allow a preliminary diagnostic evaluation and a judgement on the need for a biopsy diagnosis or excision. As our preliminary experience suggests, dysplastic nevi differ from congenital nevi by the 

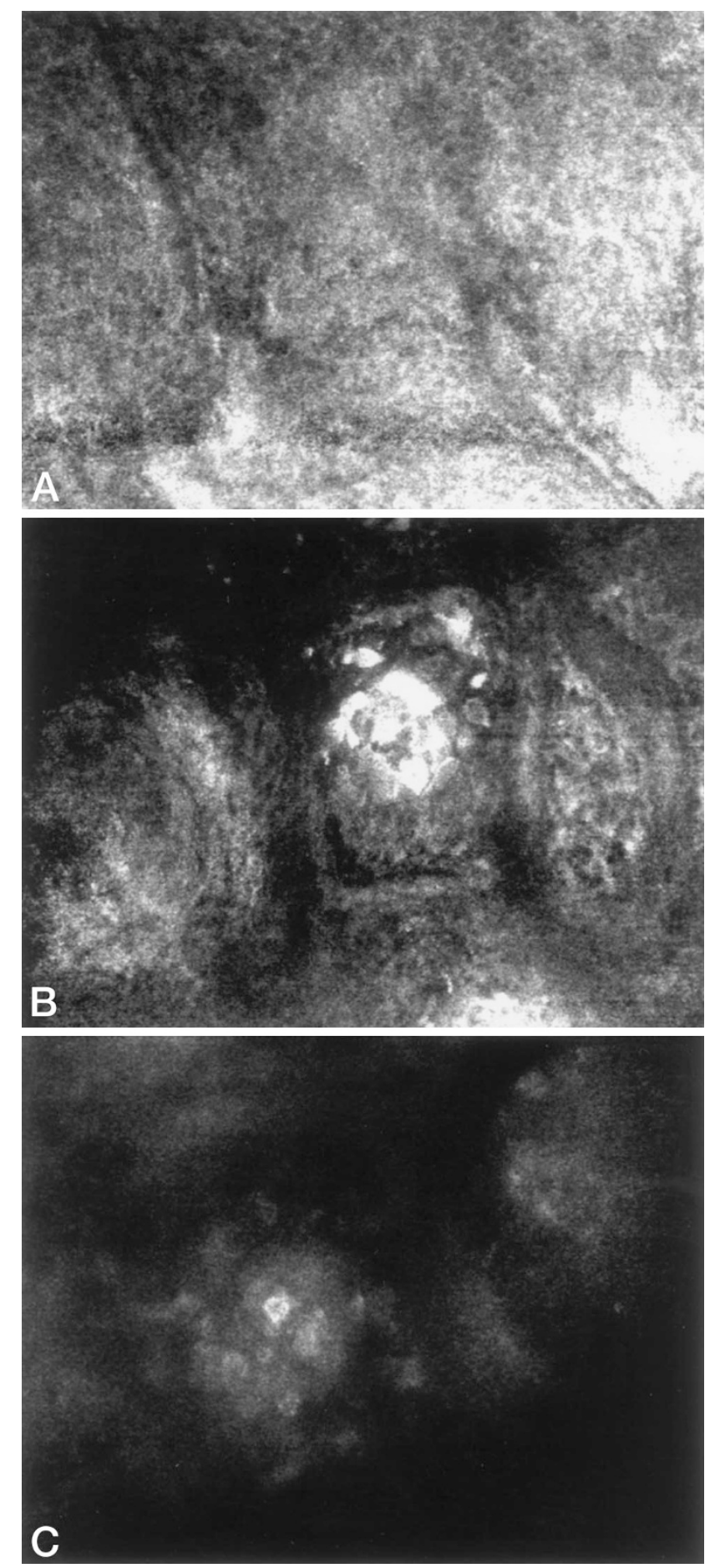

FIGURE 5. Optical sections (horizontal planes) of a compound nevus obtained by CSLM at various depths from the surface. A, level of upper spinous and granular cell layer (16 $\mu \mathrm{m}$ from the skin surface). Keratinocytes of the granular cell layer are very bright. B, level of midepidermis and lower epidermis, including dermoepidermal junction (32 $\mu \mathrm{m}$ from the surface). Dark spaces correspond to peaks of dermal papillae. C, level of dermoepidermal junction and superficial dermis (64 $\mu \mathrm{m}$ from the surface).

presence of a more irregular melanocytic growth pattern along the dermoepidermal junction. However, it is too early to judge to what extent confocal microscopy may allow a reliable classification of various nevi. A large number of melanocytic nevi need to be evaluated by CSLM to determine the sensitivity and specificity of the diagnoses that can be rendered using this technique. At its current state of technology, we are skeptical that CSLM can provide sufficient information for histologic typing of nevi. Attempts for technical improvements, however, are already underway and will likely result in better image resolution for more specific diagnoses. Nonetheless, even at its current state, a preliminary evaluation of a pigmented lesion in vivo by confocal microscopy may be of great benefit for clinicians. If a pigmented lesion is recognized as melanocytic in origin by identification of an increased number of melanocytes in the epidermis through CSLM, this is already valuable information. If a melanocytic lesion is then judged as atypical in its intraepidermal growth pattern, such as by identification of solitary melanocytes above the basal cell layer by CSLM, the clinician knows that an excision needs to be performed. Another potential application of CSLM in its current technological state is the noninvasive assessment of the perimeter of a clinically poorly definable melanoma. The use of CSLM may spare a patient multiple punch biopsies for mapping margins of a lesion before final surgical excision. We have already successfully used CSLM in this regard with cases of clinically amelanotic melanoma (19).

The main advantage of CSLM is the fact that it permits noninvasive, quasihistologic assessment of the skin at the bedside, albeit at a level of resolution inferior to that of conventional histology. Different lesions can be examined during the same office visit, and the same lesion can be examined at different points in time. Images are available within seconds, and no tissue processing is necessary. Images can be stored electronically and quickly shared for consultation. Because the evaluation of images obtained by CSLM relies on the analysis of microanatomic structures, experience in histopathology is critical for the diagnostic use of this technique.

In this report, we present for the first time in detail the morphologic features of the cellular constituents of pigmented lesions under near-infrared reflectance CSLM. This technique represents an opportunity for pathologists to expand the realm of their expertise to noninvasive image modalities and allows them to examine lesions on patients in vivo. Although at the present time the technique is predominantly used in dermatology, mucosal surfaces are also amenable to examination by CSLM, and this is already being explored. Thus, analysis of images obtained by CSLM should be of interest to all pathologists.

Acknowledgments: The authors thank Dr. J. Zavislan, Lucid, Inc., Henrietta, NY, for technical support. 
We thank Dr. S. Gonzalez, Wellman Laboratories, Department of Dermatology, Massachusetts General Hospital, for advice. We are grateful to the technical staff of the Department of Pathology for processing and staining the tissue sections.

\section{REFERENCES}

1. Grin CM, Kopf AW, Welkovich B, Bart RS, Levenstein MJ. Accuracy in the clinical diagnosis of malignant melanoma. Arch Dermatol 1990;126:763-6.

2. Sober AJ, Burstein JM. Computerized digital image analysis: an aid for melanoma diagnosis - preliminary investigations and brief review. J Dermatol 1994;21:885-90.

3. Pehamberger $\mathrm{H}$, Binder $\mathrm{M}$, Steiner A, Wolff $\mathrm{K}$. In vivo epiluminescence microscopy: improvement of early diagnosis of melanoma. J Invest Dermatol 1993;100:356-62S.

4. Rajadhyaksha M, Grossman M, Esterowitz D, Webb RH, Anderson RR. In vivo confocal scanning laser microscopy of human skin: melanin provides strong contrast. J Invest Dermatol 1995;104:946-52.

5. Rajadhyaksha M, Gonzalez S, Zavislan JM, Anderson RR, Webb RH. In vivo confocal scanning laser microscopy of human skin II: advances in instrumentation and comparison with histology. J Invest Dermatol 1999;113:293-303.

6. Gonzalez S, Gonzalez E, White WM, Rajadhyaksha M, Anderson RR. Allergic contact dermatitis: correlation of in vivo confocal imaging to routine histology. J Am Acad Dermatol 1999;40:708-13.

7. Gonzalez S, Rajadhyaksha M, Gonzalez-Serva A, White WM, Anderson RR. Confocal reflectance imaging of folliculitis in vivo: correlation with routine histology. J Cutan Pathol 1999; 26:201-5.
8. Gonzalez S, Rajadhyaksha M, Rubinstein G, Anderson RR Characterization of psoriasis in vivo by reflectance confocal microscopy. J Med 1999;30:337-56.

9. Hongcharu W, Dwyer P, Gonzalez S, Anderson RR. Confirmation of onychomycosis by in vivo confocal microscopy. J Am Acad Dermatol 2000;42:214-6.

10. Aghassi D, Anderson RR, Gonzalez S. Time-sequence histologic imaging of laser-treated cherry angiomas with in vivo confocal microscopy. J Am Acad Dermatol 2000;43:37-41.

11. Aghassi D, Anderson RR, Gonzalez S. Confocal laser microscopic imaging of actinic keratoses in vivo: a preliminary report. J Am Acad Dermatol 2000;43:42-8.

12. Aghassi D, Gonzalez E, Anderson RR, Rajadhyaksha M, Gonzalez S. Elucidating the pulsed-dye laser treatment of sebaceous hyperplasia in vivo with real-time confocal scanning laser microscopy. J Am Acad Dermatol 2000;43:49-53.

13. Gonzalez S, White WM, Rajadhyaksha M, Anderson RR, Gonzalez E. Confocal imaging of sebaceous gland hyperplasia in vivo to assess mechanism and efficacy of pulsed dye laser treatment. Lasers Surg Med 1999;25:8-12.

14. Langley RGB, Rajadhyaksha M, Dwyer PJ, Anderson RR, Sober AJ. Confocal scanning laser microscopy of pigmented skin lesions. SID conference 1996 [abstract]. J Invest Dermatol 1996;106:836.

15. Pierard GE. In vivo confocal microscopy: a new paradigm in dermatology. Dermatology 1993;186:4-5.

16. Wilson T, editor. Confocal microscopy. San Diego: Academic Press, 1990.

17. Webb RH. Confocal optical microscopy. Rep Prog Phys 1996; 59:427-71.

18. Dunn AK, Smithpeter C, Welch AJ, Richards-Kortum R. Sources of contrast in confocal reflectance imaging. Appl Opt 1996;35:3441-6.

19. Busam KJ, Hester K, Charles C, Sachs DL, Gonzalez S, Halpern A. Detection of clinically amelanotic malignant melanoma and mapping of its margins by in vivo confocal laser scanning microscopy (CSLM). Arch Dermatol 2001;137:923-9. 